NASA Technical Memorandum 107134

AIAA-96-0387

\title{
Results and Current Status of the NPARC Alliance Validation Effort
}

Charles E. Towne

Lewis Research Center

Cleveland, Ohio

and

Ralph R. Jones

Sverdrup Technology, Inc.IAEDC Group

Arnold Air Force Base, Tennessee

Prepared for the

34th Aerospace Sciences Meeting and Exhibit

sponsored by the American Institute of Aeronautics and Astronautics

Reno, Nevada, January 15-18, 1996

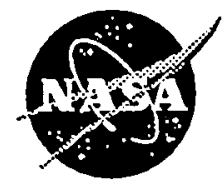

National Aeronautics and

Space Administration

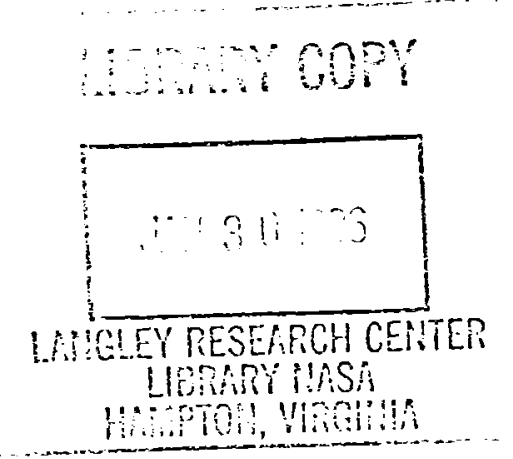




\title{
RESULTS AND CURRENT STATUS OF THE NPARC ALLIANCE VALIDATION EFFORT
}

\author{
Charles E. Towne ${ }^{1}$ \\ NASA Lewis Research Center \\ Cleveland, Ohio \\ Ralph R. Jones ${ }^{2}$ \\ Sverdrup Technology, Inc./AEDC Group \\ Arnold Engineering Development Center \\ Amold Air Force Base, Tennessee
}

\begin{abstract}
The NPARC Alliance is a partnership between the NASA Lewis Research Center (LeRC) and the USAF Arnold Engineering Development Center (AEDC) dedicated to the establishment of a national CFD capability, centered on the NPARC Navier-Stokes computer program. The three main tasks of the Alliance are user support, code development, and validation. The present paper is a status report on the validation effort. It describes the validation approach being taken by the Alliance. Representative results are presented for laminar and turbulent flat plate boundary layers, a supersonic axisymmetric jet, and a glancing shock/turbulent boundary layer interaction. Cases scheduled to be run in the future are also listed. The archive of validation cases is described, including information on how to access it via the Internet.
\end{abstract}

\section{Introduction}

The NPARC Alliance is a partnership between the NASA Lewis Research Center (LeRC) and the USAF Arnold Engineering Development Center (AEDC) dedicated to the establishment of a national computational fluid dynamics (CFD) capability, centered on the NPARC computer program. The NPARC code is based on the PARC code, which by 1993 was being widely used by a variety of government, industrial, and academic institutions. Several of these users had approached both LeRC and AEDC about establishing a formal organization for the further support, development, and validation of the PARC code. The NPARC Alliance was established in 1993 in response to these requests.

The three main tasks of the NPARC Alliance are

1. Senior Member, AIAA

2. Associate Fellow, AIAA

This paper is declared a work of the U. S. Government and is not subject to copyright protection in the United States. user support, program development, and validation. The Support Team coordinates the release of the software, assists users in its application, resolves bug reports and other problems, and manages the various communication channels between users and the Alliance. The Development Team establishes directions for future development of the NPARC code, develops enhancements, and incorporates improvements contributed by other developers. The Validation Team is responsible for validating the NPARC code for a wide range of flow parameters and geometric configurations, and for establishing an archive of cases that can be accessed by the NPARC community to support independent assessment of the code's capabilities.

This paper describes in general the approach being taken in the NPARC validation effort. Representative results are presented from validation and example cases already run, and cases scheduled to be run in the future are described. Finally, the validation archive is discussed, including information on how to access it via the Internet. Additional details on all the cases presented here may be found in the documentation available in the NPARC validation archive.

\section{Validation Approach}

The validation effort is intended to establish the basis upon which confidence in results produced by NPARC is founded, and the practical limits on the accuracy of predictions of flow phenomena pertinent to propulsion-oriented flows. Such confidence can only be achieved through a continuous process of careful application of the code to a wide range of "unit" and "configuration-oriented" problems and complete documentation of results. Here, "unit" refers to problems focusing on a single phenomenon and simple geometries, whereas "configuration-oriented" refers to problems 
which focus on geometries and flows more representative of typical propulsion system components.

A wide variety of propulsion-oriented validation problems representing a mix of flows have been identified as candidates for validation cases. Cases are run to determine the strengths and weaknesses of the NPARC code for a variety of geometric configurations, and over a range of flow parameters. Computed results are compared with benchmark-quality experimental data, wellaccepted computational results, and/or analytic solutions.

Initial validation efforts have focused on "unit" or single phenomenon problems, and provide both the initial entries into the validation archive as well as serve as models for future validation case execution and documentation. The following list indicates the type of "unit" problems that may be used for NPARC validation:

- Flat plate boundary layers, including heat and mass transfer

- Falkner-Skan flows

- Curved-wall boundary layers

- Free shear layers

- Flow past simple bodies (e.g., a cylinder, sphere, or cone)

- Pressure-driven secondary flows

- Entrainment flows

- Shock/boundary layer interactions

- Separated flows

- Vortex flows

- Wake flows

- Forward and backward facing steps

The longer term activities are aimed at maintaining a process of continuous validation case execution with a goal of a minimum of 3-4 validation cases executed per year. Increased emphasis is placed on "configurationoriented" problems such as:

- Propulsive nozzle/afterbody

- Airbreathing (axisymmetric and 2-D)

- Rocket (single and multiple nozzles)

- Airfoil cascade

- Diffusing duct

- Propulsion system inlet (subsonic and supersonic)

- Ejector nozzle

\section{Model Validation Cases}

The term "validation" has been used in a variety of ways in the literature. For this effort, we are guided by the following definition, adapted from one given by Mehta (1990):
A code is said to be validated if the following conditions are met: (1) a comparison of computed results with detailed surface and flow field experimental data and/or other well-accepted solutions shows that the code is able to accurately model the critical physics of the flow; (2) the accuracy and limitations of the experimental data are known and understood; and (3) the accuracy and limitations of the code's numerical algorithms, grid density effects, convergence effects, and physical basis are known and understood. The range of applicability of the validated code depends on the range of flow parameters and/or geometric configurations for which the code has been validated.

Of course, in practice the accuracy and limitations of the experimental data and the computational results cannot be fully "known and understood." In addition, the degree to which the code must "accurately model the critical physics of the flow" will depend on how the results are to be used. These factors will inevitably introduce some blurring of the line between the states of validation and non-validation. Nevertheless, this definition does serve to provide the necessary philosophy that guides the validation effort. NPARC validation cases which attempt to meet this strict standard are termed model (i.e., ideal) cases.

Model cases are run to determine the strengths and weaknesses of the NPARC code for a variety of geometric configurations, and over a range of flow parameters. The Validation Team coordinates with the Development Team to correct weaknesses in the code, and to anticipate validation needs relative to on-going and planned development work.

The accuracy and limitations of the code are investigated by examining the sensitivity of the results to various input options such as mesh density, turbulence model, and artificial viscosity model. The validation effort is expected to be an on-going activity and, through the NPARC Association, users are encouraged to propose candidate validation problems and submit documentation and results from independent validation efforts.

Each model validation case is documented, in a consistent format, as part of the validation archive. A consistent format for the documentation of validation cases is necessary to ensure an adequately thorough representation of a given case and to permit a comparison of conclusions drawn from a variety of cases. The documentation highlights the primary focus and pertinent findings for each case, and includes sufficient detail to allow independent repetition of each case. A Validation Abstracts document will be developed summarizing all 
the model validation cases to compliment the detailed information in the validation archive.

\section{Example Validation Cases}

Example cases are established and documented in coordination with the Support team. There are two primary goals which the example validation cases are designed to meet. The first is to provide users with quick, but limited validation of the NPARC software over a wide range of flows. These validation cases are indicative of the capabilities of the flow simulation program, but do not meet the definition of a model validation case in that they do not examine the sensitivity of the results to various input options. The second goal of the example cases is to provide the new user with clear examples of how to properly setup and execute the NPARC code for a variety of geometries and flow conditions.

The documentation for the example cases includes: a description of the problem being solved; a description of the computational mesh and initial condition files, including, where appropriate, listings of codes and input used to create these files; a discussion and listing of the input used to run the NPARC code, including typical job control commands and the NAMELIST input file; and a discussion of the computed results and convergence history. The documentation is automatically provided with the NPARC code as part of the NPARC User's Guide (NPARC Alliance, 1994) and/or as a separate document. It is also available as part of the validation archive.

\section{Check Cases}

Check cases will be established to judge the functionality of a newly installed and/or modified code. These will be developed, maintained, and documented in conjunction with the Development Team. The primary intent of the check cases is to provide the Development team with a tool to ensure the integrity of all mechanical aspects of code operation. At least one of these cases will be an installation check case that is intended for use by new recipients of the NPARC code to verify that the code has been properly installed on their computer system.

The documentation for the check cases will be included as part of the Developer's Guide, and will also be available as part of the validation archive.

\section{Flat Plate Boundary Layer}

\section{Laminar Flow}

One of the first model validation cases run with NPARC was "incompressible" laminar flow past a flat plate. A free stream Mach number of 0.1 was used to minimize compressibility effects, and allow comparison between the computed results and the exact incompressible Blasius boundary layer solution. The Reynolds number $R e_{x}$, based on the free stream velocity and distance from the leading edge, ranged from 0 to 200,000 .

Several cases were run for this problem to examine the effects of various parameters on the convergence rate and the final results. These included the effects of: (1) mesh resolution; (2) outer boundary height; (3) inflow boundary conditions; (4) inlet plane location; (5) initial conditions; (6) artificial viscosity; and (7) using the $2 \mathrm{D}$ or $3 \mathrm{D}$ code. All of the cases were run 12,000 time steps, with the default value of 2 for IVARDT. DTCAP was set equal to 5.0 for the first 3,000 steps, 10.0 for the next 3,000 steps, and 20.0 for the last 6,000 steps. For most of the cases, the results were essentially the same, and agreed very well with the Blasius boundary layer solution. Changing the outer boundary height, however, did affect the computed values of the normal velocity component.

Three cases were run to investigate the effect of the outer boundary height. All used 126 grid points in the $x$ direction, evenly distributed between $x=-0.25$ and $x=1.0$, where $x=0.0$ corresponds to the plate leading edge. For the $y$ direction, a Blasius coordinate transformation was used, with

$$
\eta=y\left(\frac{u_{e}}{2 v x}\right)^{1 / 2}
$$

where $\eta$ is the Blasius similarity coordinate, $u_{e}$ is the free stream velocity and $v$ is the kinematic viscosity. First the $\eta$ coordinates were computed, with 21 points evenly distributed between $\eta=0.0$ and $\eta=4.0$. Above $\eta=4.0$, which corresponds approximately to the boundary layer edge in the Blasius solution, the $\eta$ coordinate was stretched geometrically using

$$
\eta_{k}=\eta_{k-1}+r\left(\eta_{k-1}-\eta_{k-2}\right)
$$

where $k$ is the index in the $y$ direction. For $x \geq 0.25$, the $y$ coordinates were computed from Equation (1). For $x<0.25$, the $y$ coordinates were set to those at $x=0.25$ to prevent the height of the computational domain from approaching zero at the plate leading edge.

For the first case, 24 points were added above $\eta=4.0$, which gave a maximum $k$ value of 45 and $\eta_{45}=23.47$. For the second case, 32 points were added, which gave $\eta_{53}=48.25$. Both of these cases used a stretching factor of $r=1.1$. For the third case, a stretching factor of $r=1.05$ was used, and 65 points were added, which gave $\eta_{86}=99.93$. (Initially, $r=1.1$ 
was also used in the third case, but the solution was unstable. It was thought that the large grid spacing in the $y$ direction far away from the plate may have been a factor in causing the instability, and lowering the stretching factor solved the problem.)

Five boundary segments were used: (1) the upstream inflow boundary; (2) the downstream outflow boundary; (3) the symmetry plane on the lower boundary upstream of the plate; (4) the flat plate itself; and (5) the upper free stream boundary. The boundary conditions used, including the code number in the NPARC input, are summarized in the following table.

\begin{tabular}{|l|l|r|}
\hline \multicolumn{1}{|c|}{ Boundary } & \multicolumn{1}{|c|}{ Condition } & Code \# \\
\hline Upstream & Fixed conditions & -10 \\
Downstream & Subsonic outflow & 0 \\
Symmetry plane & Symmetry & 50 \\
Flat plate & No-slip adiabatic & 60 \\
Upper & Free stream & 7 \\
\hline
\end{tabular}

The initial conditions were uniform flow at $M=0.1$ for all three cases.

The second-order artificial viscosity coefficient DIS2 was kept at its default value of 0.25 for the first 6,000 time steps, then lowered to 0.0 for the last 6,000 time steps. The fourth-order coefficient DIS4 was kept at its default value of 0.64 for all 12,000 time steps.

The computed $u$ - and $v$-velocity profiles in the boundary layer region for the three cases are shown in Figures 1 and 2. These profiles are at $x / L=0.75$, and are after 12,000 time steps. Also shown is the exact Blasius boundary layer solution (White, 1974). The $u$ velocity results are nearly identical for all three cases. However, the $v$-velocity profiles show significantly better agreement with the Blasius solution when the height of the free stream boundary is increased. (Note, however, that in the Blasius similarity solution the $v$ velocity is normalized differently than the $u$-velocity, and is actually much smaller in magnitude than the $u$ velocity. In the Blasius solution, $R e_{x}^{1 / 2} v / u_{e}$ approaches a constant value of 0.8604 far from the plate. At this $x$ location on the plate $(x / L=0.75)$, this corresponds to $v / u_{e}=0.00222$.)

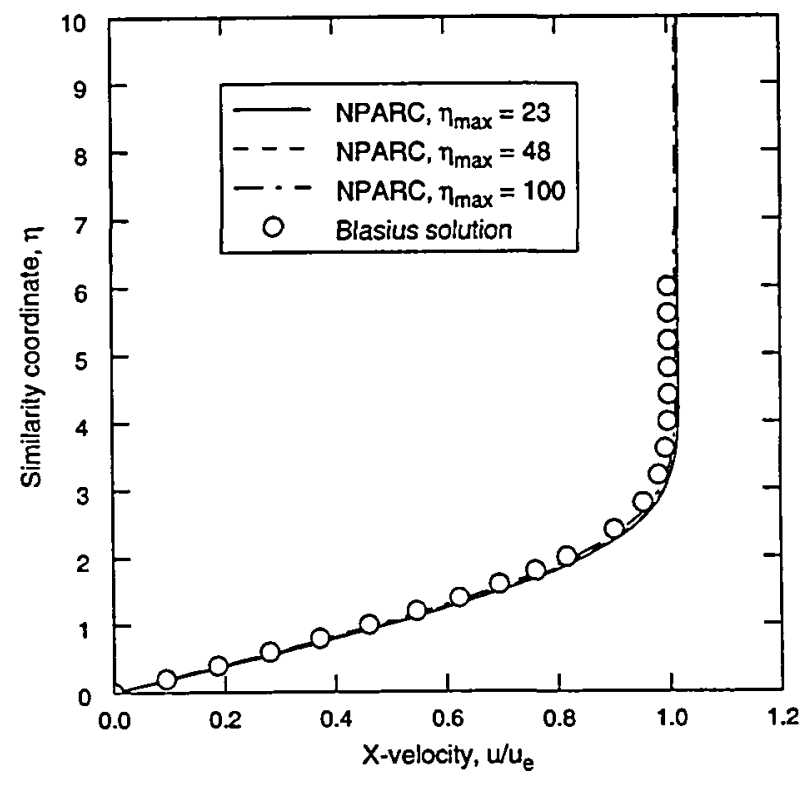

Figure 1. Effect of free stream height on $u$-velocity profiles for laminar flow past a flat plate.

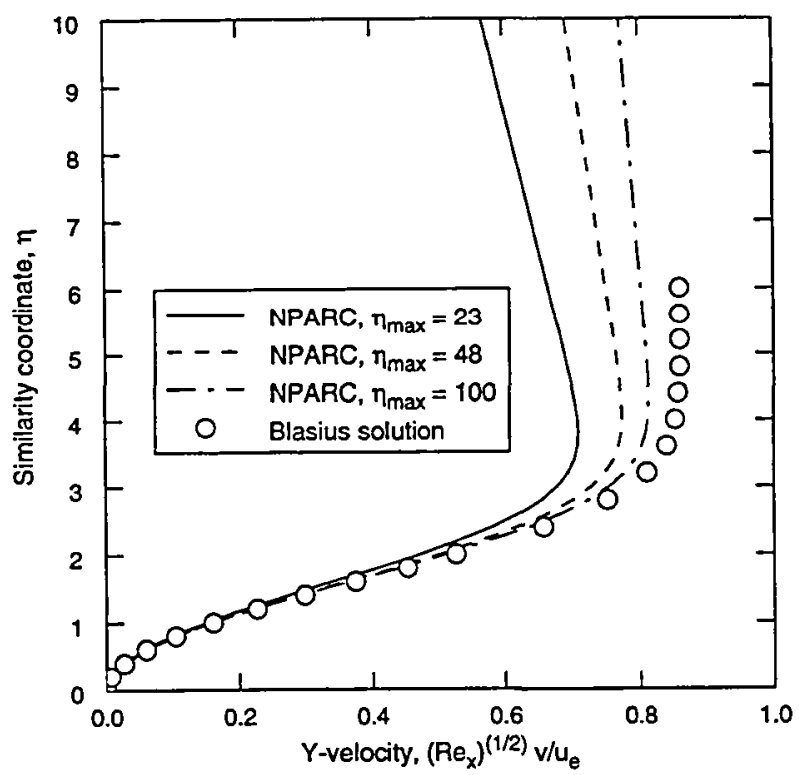

Figure 2. Effect of free stream height on $v$-velocity profiles for laminar flow past a flat plate.

\section{Turbulent Flow}

Another basic model validation case run with NPARC was turbulent flow past a flat plate. A free stream Mach number of 0.2 was used to minimize compressibility effects, and allow comparison between the computed results and incompressible experimental data. The Reynolds number $R e_{x}$, based on the free stream velocity and distance from the leading edge, ranged from 0 to $1 \times 10^{7}$. 
As in the laminar flow problem, several cases were run to examine the effects of various parameters on the convergence rate and the final results. These included the effects of: (1) mesh resolution; (2) the $y^{+}$value at the first grid point away from the wall; (3) turbulence model; (4) inflow boundary conditions; (5) inlet plane location; (6) artificial viscosity; and (7) the time step selection option. In general, the computed results agreed very well with experimental data. The most significant differences between calculations were due to inadequate mesh resolution, too-large $y^{+}$values, and the choice of turbulence model. As an example, the following discussion presents results for different $y^{+}$values, with the Baldwin-Lomax (1978) turbulence model.

The computational mesh was generated algebraically. Grid points in the $x$ direction were packed near the leading edge of the plate, and in the $y$ direction near the plate surface, using a Roberts transformation (Roberts, 1971).

For the $x$, or $j$, direction, 126 points were used, with the leading edge at $x=0.0$ corresponding to $j=26$. The computational domain extended from $x_{1}=-1.0352$, upstream of the leading edge, to $x_{126}=10.0$.

For the $y$, or $k$, direction, 76 points were used, with 51 in the boundary layer. The boundary layer thickness was estimated as follows (Daily and Harleman, 1966):

$$
\delta=\frac{0.38 x}{R e_{x}^{1 / 5}}
$$

Different amounts of grid packing were used within the boundary layer to give different $y^{+}$values at the first grid point away from the wall. For $y>\delta$, the $y$ coordinates were stretched geometrically using

$$
y_{k}=y_{k-1}+r\left(y_{k-1}-y_{k-2}\right)
$$

The stretching factor $r$ was 1.1. For $j<36$, the $y$ coordinates were set to those at $j=36$ to prevent the height of the computational domain from approaching zero at the plate leading edge.

It should be noted that since the number of points in the boundary layer was fixed, tighter spacing near the wall meant larger spacing near $y=\delta$. Since there were 25 points outside the boundary layer for all the cases, the height of the outer boundary varied with the degree of packing near the wall. Also, since $y^{+}$depends on the computed flow field in addition to the physical $y$ coordinate, the $y^{+}$values did not scale exactly with the $y_{2}$ values. The values of some of these grid-related parameters are shown in the following table.

\begin{tabular}{|c|c|c|r|}
\hline Case & $y_{2} / \delta$ & $y^{+}$ & \multicolumn{1}{|c|}{$y_{\max } / \delta$} \\
\hline 8 & $0.82 \times 10^{-4}$ & $0.0-0.5$ & 10.070 \\
1 & $1.64 \times 10^{-4}$ & $0.0-1.0$ & 9.216 \\
5 & $3.33 \times 10^{-4}$ & $0.0-1.9$ & 8.329 \\
6 & $6.56 \times 10^{-4}$ & $0.0-3.4$ & 7.468 \\
7 & $13.11 \times 10^{-4}$ & $0.0-5.5$ & 6.578 \\
24 & $26.29 \times 10^{-4}$ & $0.0-8.2$ & 5.678 \\
\hline
\end{tabular}

As in the laminar case, five boundary segments were used: (1) the upstream inflow boundary; (2) the downstream outflow boundary; (3) the symmetry plane on the lower boundary upstream of the plate; (4) the flat plate itself; and (5) the upper free stream boundary. The boundary conditions were the same as in the laminar case. The initial conditions were uniform flow at $M=0.2$.

Cases 1, 5-7, and 24 were run 14,000 time steps with DTCAP $=5.0$. Because of the tighter mesh spacing, however, case 8 required more iterations and a smaller time step for stability. It was first run 2,000 steps with DTCAP $=5.0$ to get past the starting transient. During this initial run, the parameter PCQMAX, which was left at its default value of 10.0 , controlled the actual time step size. The value of DTCAP was then lowered to 1.0 for 2,000 iterations, then raised to 2.0 for the rest of the computation. A total of 28,000 iterations were taken for case 8 .

The computed $u$-velocity profiles for cases $1,6-8$, and 24 are shown in Figure 3, along with the experimental data of Weighardt (Coles, D. E., and Hirst, E. A., 1968). The results for the three cases with $y^{+}<3.4$ are essentially the same, but differences can be seen for cases with larger $y^{+}$values. As $y^{+}$increases, the velocity profiles tend to become less steep.

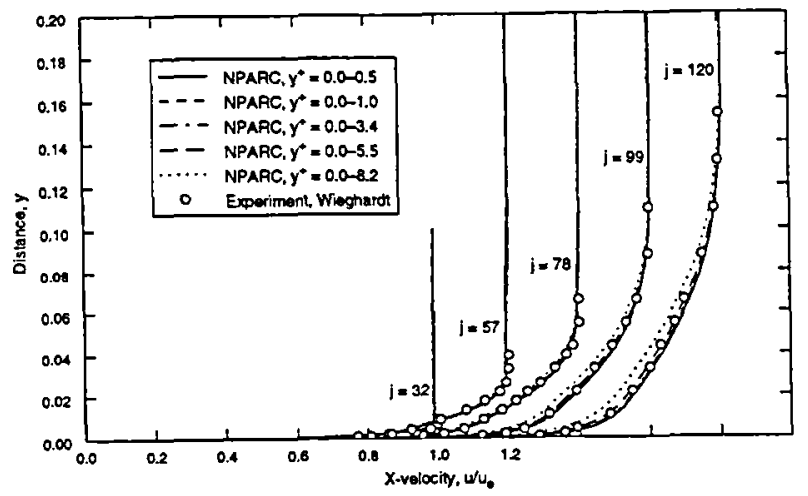

Figure 3. Effect of initial $y^{+}$on $u$-velocity profiles for turbulent flow past a flat plate, Baldwin-Lomax model.

The computed skin friction coefficients are compared with the experimental data of Wieghardt, and 
with values from two different correlation formulas (White, 1974), in Figure 4. The results for the two cases with $y^{+}$everywhere below 1.0 are essentially identical. As $y^{+}$increases, the skin friction decreases, which is consistent with the velocity profile results shown in Figure 3.

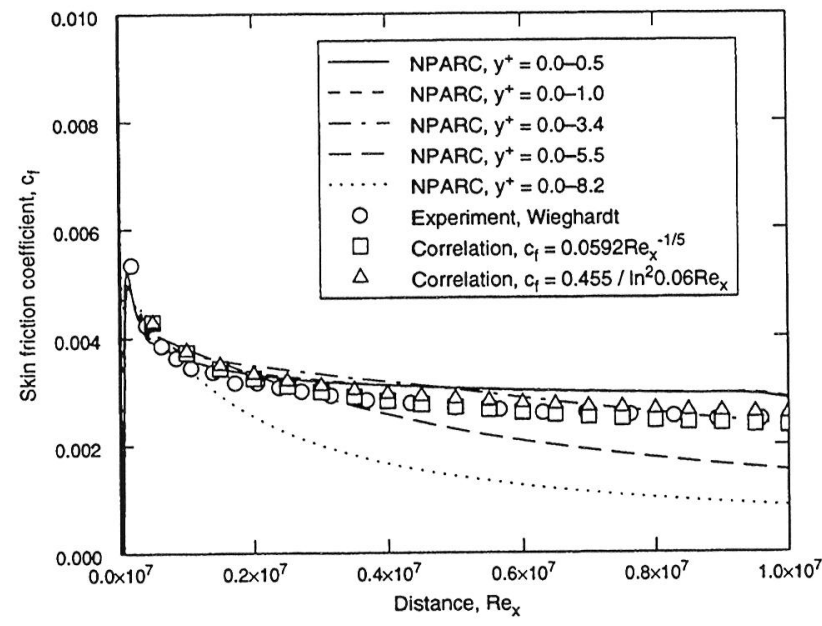

Figure 4. Effect of initial $y^{+}$on skin friction distribution for turbulent flow past a flat plate, Baldwin-Lomax model.

\section{Supersonic Axisymmetric Jet}

Another model validation case currently being computed is the turbulent supersonic axisymmetric jet flow studied experimentally by Eggers (1966). One of the runs was used as an example validation case, and some of the results from that calculation are presented here. This case is described in detail in the NPARC User's Guide (NPARC Alliance, 1994).

The jet is produced by an axisymmetric convergentdivergent nozzle. This study focuses primarily on the development of the jet downstream of the nozzle exit, although care is taken to properly model the flow within the nozzle. The basic geometric configuration is shown in Figure 5.

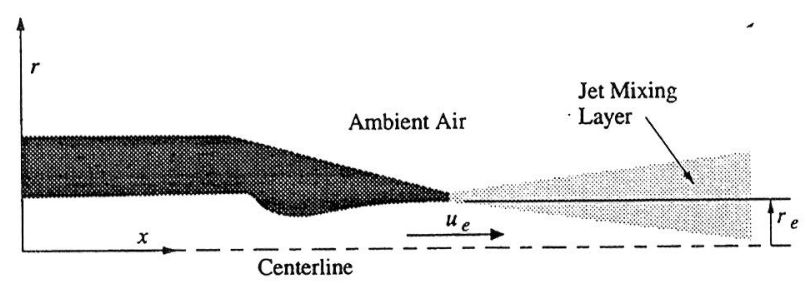

Figure 5. Geometric configuration for axisymmetric jet flow.

The nozzle total pressure and temperature were 162.2 psia and $525^{\circ} \mathrm{R}$, respectively, and these values were selected as the reference values. The reference length $X_{r}$ was $1 \mathrm{ft}$, giving a reference Reynolds number $R e_{r}=a_{r} X_{r} / v_{r}$ of $78.8 \times 10^{6}$, where $a_{r}$ and $v_{r}$ are the speed of sound and kinematic viscosity evaluated at the reference conditions. The nozzle was operated at a pressure ratio of 0.09063 , corresponding to perfect expansion with an exit Mach number of 2.22. The nozzle exit radius was 0.5035 in.

The computational mesh was generated using GRIDGEN (Steinbrenner, J. P., Chawner, J. R., and Fouts, C. R., 1990). The mesh consisted of 336 points in the $x$, or $j$, direction, and 107 points in the $r$, or $k$, direction. In the nozzle interior, there were 188 and 59 points in the $x$ and $r$ directions respectively. The computational domain extended approximately 145 nozzle exit radii downstream of the nozzle exit. In the radial direction, the grid extended approximately 12 nozzle exit radii at the nozzle exit plane, and approximately 48 nozzle exit radii at the exit of the computational domain. The grid in the region of the nozzle is shown in Figure 6. For clarity, only every fourth grid line in the $x$ direction and every sixth grid line in the $r$ direction is shown.

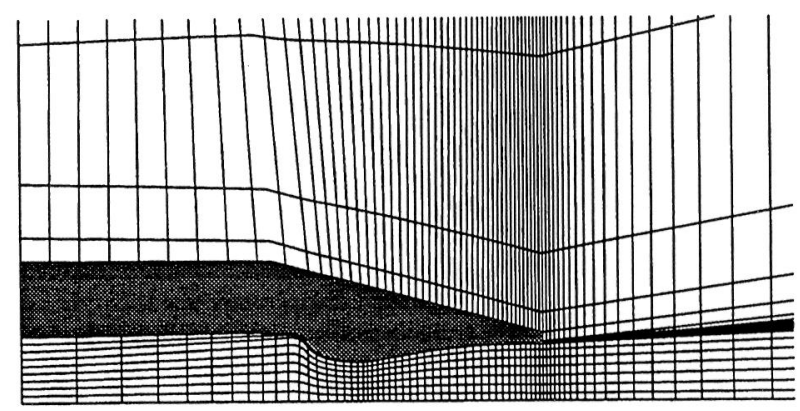

Figure 6. Computational mesh for axisymmetric jet flow.

As mentioned earlier, care was taken in constructing the grid interior to the nozzle to ensure adequate representation of the nozzle exit flow. It was found through preliminary inviscid studies that the quality of flow at the exit of the nozzle was highly dependent on the axial spacing of the grid in the diverging section. In particular, adequate nozzle wall resolution was required to minimize the generation of a series of compression waves from the discrete representation of the wall geometry. For this problem, 110 points in the $x$ direction in the diverging section were found to be adequate.

As an additional note, since the original report by Eggers provides no details as to how the nozzle was connected to the supply reservoir, the inviscid study also focused on nozzle entrance effects. The nozzle was assumed to draw from both a constant area duct as well as an "infinite" radius volume. This study 
indicated no effect of the reservoir geometry on nozzle wall pressure distribution downstream of the throat. Thus, for the purpose of this example case, the nozzle was assumed attached to a constant area duct.

Since the focus of this example is the development of the mixing region downstream of the nozzle exit, grid resolution within the jet shear layer throughout its development region was a principal concern. The minimum radial grid packing is driven by the shear layer thickness at the nozzle exit. To estimate this thickness and also provide guidance relative to the radial grid spacing adjacent to the nozzle wall, the finite difference boundary layer code BLAYER (Hodge, B. K., and Adams, J. C., Jr., 1978) was used to estimate the boundary layer growth within the nozzle. This required as input a nozzle wall pressure distribution, which was taken from the nozzle entrance effects study. From these results, the physical grid spacing required to maintain at least one grid point in the laminar sublayer (i.e., $y^{+} \leq 1$ ) was estimated to be $1 \times 10^{-5}$ inches. The $k$-constant grid line emanating from the nozzle exit lip (i.e., $k=59$ ) was positioned to approximate the center of the jet shear layer based upon Eggers' data, and provided the location about which radial grid packing for shear layer resolution was centered. This radial packing was gradually relaxed toward the flow domain exit boundary where the radial grid spacing was uniform between the centerline and the $k=59$ grid line and gradually increased from $k=59$ to the outer boundary at $k=107$. The clustering function used was the default hyperbolic tangent function in GRIDGEN. Points were also packed in the $x$ direction near the nozzle exit lip, where the first axial grid spacing downstream of the lip was $8 \times 10^{-3}$ inches.

A total of eight boundary segments were used in the NPARC calculation: (1) the nozzle inflow boundary; (2) the downstream outflow boundary; (3) the nozzle exit lip; (4) the upstream free field inflow boundary (exterior to the nozzle); (5) the centerline; (6) the upper freestream inflow boundary; (7) the nozzle interior wall; and (8) the nozzle exterior wall. The boundary conditions used, including the code number in the NPARC input, are summarized in the following table. ${ }^{3}$

\begin{tabular}{|l|l|c|}
\hline \multicolumn{1}{|c|}{ Boundary } & \multicolumn{1}{|c|}{ Condition } & Code \# \\
\hline Nozzle inflow & Subsonic inflow & 0 \\
Downstream outflow & Subsonic outflow & 0 \\
Nozzle exit lip & Slip wall & 50 \\
Upstream free field & Subsonic inflow & 0 \\
$\quad$ inflow & & \\
Centerline & Axis of symmetry & 51 \\
Upper freestream & Subsonic inflow & 0 \\
$\quad$ inflow & & \\
Nozzle interior wall & No-slip adiabatic & 60 \\
Nozzle exterior wall & Slip wall & 50 \\
\hline
\end{tabular}

The initial conditions were established by setting the temperature and pressure equal to the ambient values, and each velocity component to zero for all points in the computational domain except those interior to the nozzle. There, total conditions were set to the supply values, and velocities were initialized by linearly interpolating in the $x$ direction using the $j$ index, from $M=0.3$ at the nozzle inflow plane to a $M=2.22$ at the nozzle exit plane.

A total of 10,000 time steps were taken, with the default value of 2 for IVARDT. DTCAP was set to 5.0 for the first 1500 steps, and 0.4 thereafter. For the entire solution, the maximum change allowed in pressure or density over a time step was $25 \%$. The artificial viscosity coefficients DIS2 and DIS4 were left at their default values of 0.25 and 0.64 for the first 1500 steps. DIS2 was lowered to 0.12 for the next 3500 steps, and 0.0 thereafter. The problem was run inviscidly for the first 1000 steps to quickly develop the initial plume characteristics. A combination Baldwin-Lomax (1978) and Thomas (1979) algebraic turbulence model was used for the next 1100 steps to initialize the turbulent quantities. The Chien $k-\varepsilon$ model (1982) was used for the rest of the calculation.

Figure 7 presents the computed nozzle centerline velocity distribution compared with the measurements of Eggers (1966). The local jet centerline velocity is nondimensionalized by the nozzle exit velocity and distances are measured relative to the nozzle exit station and are scaled by the nozzle exit radius.

3. During problem start-up, the nozzle interior wall was actually specified as a slip wall (i.e., code \#50), then changed to a noslip adiabatic wall after 1000 time steps. 


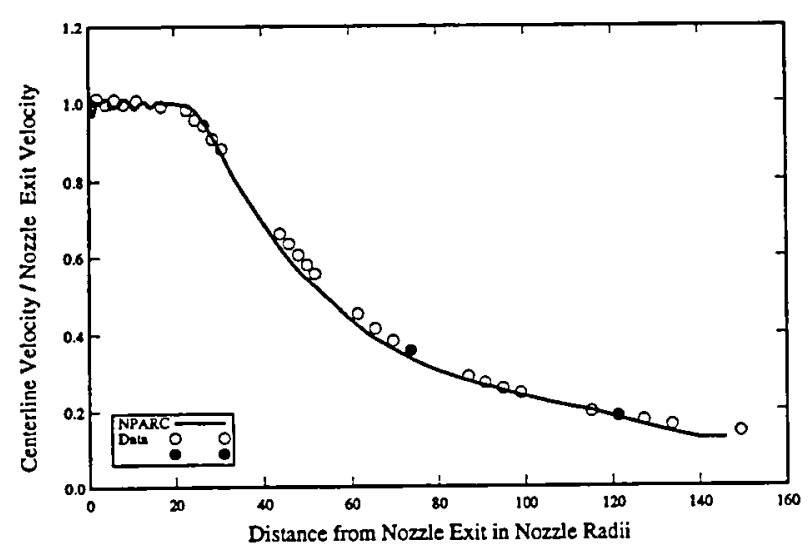

Figure 7. Nozzle centerline velocity.

The computed centerline velocity agrees well with the experimental data. As can be seen the figure, closure of the jet core occurs at approximately 20 radii. For reference, from the calculations the nozzle centerline velocity first reaches a subsonic value at approximately 45 radii and flow conditions become "incompressible" (i.e., the centerline Mach number drops below 0.3) at approximately 120 radii.

In Figures 8 and 9 , the computed velocity profiles are compared with experimental data at two locations downstream of the nozzle exit. The experimental data were taken at $x / r_{e}=73.80$ and 121.30 . These positions are indicated by the filled circles in Figure 7. The computational results are at $x / r_{e}=73.67$ and 121.46 , which correspond to the $j$ indices closest to the experimental data locations.

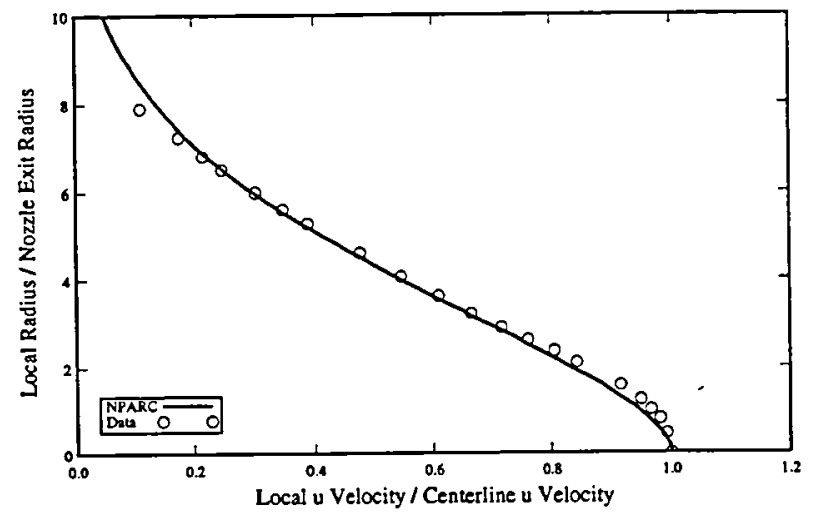

Figure 8. Velocity profile at $x / r_{e}=73.8$.

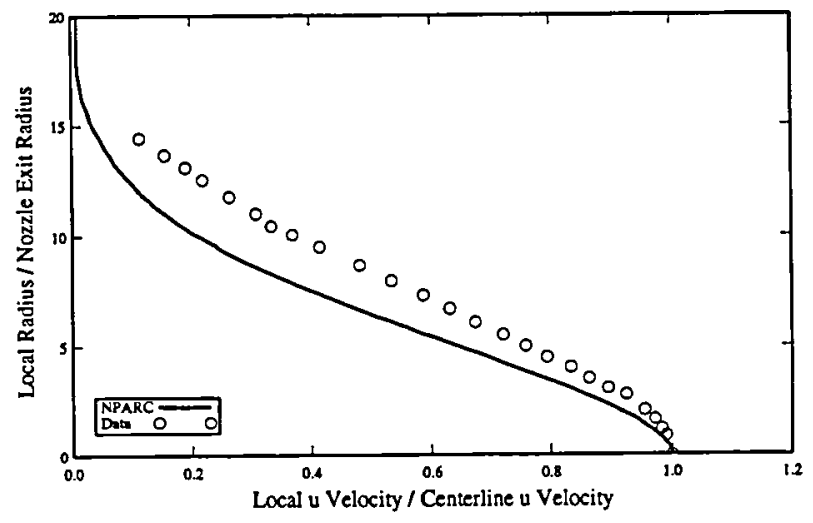

Figure 9. Velocity profile at $x / r_{e}=121.3$.

The velocity profile at $x / r_{e}=73.80$ agrees well with the experimental data, but the profile at $x / r_{e}=121.3$ does not agree as well. This may be due to the influence of the extrapolation boundary condition used at the nearby exit plane on the computed results in this region. In addition, the $x$-momentum flux for this case was continuing to decrease when the calculation was stopped (NPARC Alliance, 1994), and continuing the calculation may improve the agreement.

\section{Glancing Shock/Boundary Layer Interaction}

Another model validation study currently underway is the interaction of a glancing shock wave with a turbulent boundary layer on a flat plate. One of the cases is being used as an example validation case, and some of the results from that calculation are presented here.

The glancing shock/turbulent boundary layer interaction is produced by a Mach 3.0 flow past a sharp $10^{\circ}$ wedge, or fin, mounted on a flat plate. Figure 10 shows the geometric configuration and the outline of the computational domain. The location of the inviscid shock is also shown in the figure, with a shock angle of $29.5^{\circ}$. This flow has been studied experimentally by Kim, et. al. $(1990,1991)$, and tabulated experimental values of skin friction coefficient, surface flow angle, and surface static pressure have been published by Settles and Dodson (1991). The Reynolds number in the experiment, based on free stream conditions, was $6.19 \times 10^{7} / \mathrm{m}$, and the free stream total pressure and temperature were 827 $\mathrm{kPa}$ and $294 \mathrm{~K}$, respectively. 


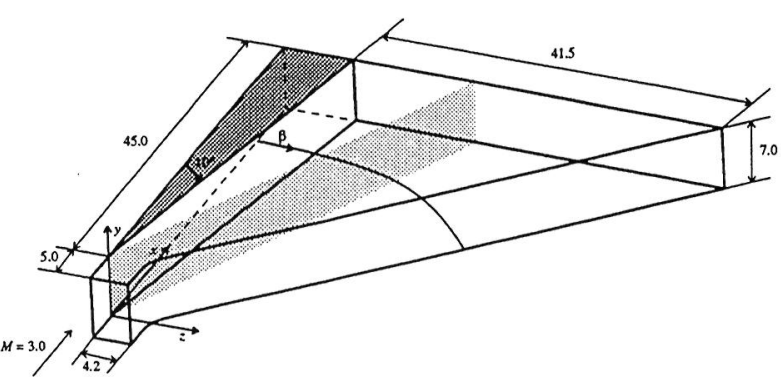

Figure 10. Geometric configuration for the glancing shock/turbulent boundary layer interaction.

Cartesian coordinates are defined with the origin at the fin leading edge, and the flat plate lies in the $x-z$ plane. The measured boundary layer thickness $38 \mathrm{~mm}$ upstream of the fin leading edge was $3.02 \mathrm{~mm}$, and this is used as the reference length. Lengths shown in the figure are non-dimensional. The leading edge of the flat plate was at $x=-71.523$.

In the experiments, data were taken along a circular arc of radius $R$, centered at the fin leading edge. For the skin friction coefficient and surface flow angle $R=37.85$, and for the surface static pressure $R=33.64$. The angular location along this arc is denoted by $\beta$, as shown in Figure 10 .

The 3-D computational mesh was generated algebraically. In the $x$ direction, 51 points were used, evenly spaced. In the $y$ direction, normal to the flat plate, the first interior point was placed at the location corresponding to $y^{+}=0.5$, where $y^{+}$was computed assuming the skin friction coefficient $c_{f}=0.00152 .{ }^{4}$ Points were then added, stretched geometrically using

$$
y_{k}=y_{k-1}+r_{y}\left(y_{k-1}-y_{k-2}\right)
$$

The stretching factor $r_{y}$ was 1.2. Equation (5) was used until

$$
\Delta y=y_{k}-y_{k-1}>(\Delta y)_{\max }
$$

where $(\Delta y)_{\max }=2000$. Above this point an evenly spaced mesh was used. The same $y$-mesh was used for all $x$ and $z$. A similar procedure was used in the $z$ direction, intersecting the fin. At the experimental measurement station for static pressure, $R=33.64$, the first interior point was placed at the location corresponding to $z^{+}=0.5$, where $z^{+}$was computed assuming $c_{f}=0.002 .{ }^{5}$ Points were then added, stretched geometrically by the factor $r_{z}=1.5$, until the spacing $\Delta z>(\Delta z)_{\max }$, where $(\Delta z)_{\max }=5000$. Beyond this point

4. This is the experimental value at the incoming boundary layer measurement station, $38 \mathrm{~mm}$ upstream of the fin leading edge.

5. This is a rough estimate of the value on the flat plate, near the fin, based on the experimental data along the data measurement radius shown in Figure 10. an evenly spaced mesh was used. This $z$-distribution was then repeated, proportionally, for all $x$ and $y$. Near the $x=0$ station the outer $z$ boundary, which would otherwise have had a slope discontinuity corresponding to the fin leading edge, was smoothed using a fifth-order polynomial. The sharp fin leading edge at the inner $z$ boundary, of course, was retained.

Seven boundary segments were used: (1) the upstream inflow boundary; (2) the downstream outflow boundary; (3) the flat plate surface; (4) the upper free stream boundary; (5) the symmetry plane at $z=0$ upstream of the wedge; (6) the wedge surface; and (7) the outer $z$ boundary. The boundary conditions used are summarized in the following table.

\begin{tabular}{|l|l|r|}
\hline \multicolumn{1}{|c|}{ Boundary } & \multicolumn{1}{|c|}{ Condition } & Code \# \\
\hline Upstream & Fixed conditions & -10 \\
Downstream & Extrapolation & 3 \\
Flat plate & No-slip adiabatic & 60 \\
Upper & Extrapolation & 3 \\
Symmetry plane & Symmetry & 50 \\
Wedge & No-slip adiabatic & 60 \\
Outer & Extrapolation & 3 \\
\hline
\end{tabular}

The conditions at the upstream inflow boundary were computed by interpolation from a 2-D NPARC calculation of the turbulent boundary layer flow past the flat plate upstream of the wedge. The initial flowfield was set equal to the flow at this upstream boundary.

The default Baldwin-Lomax algebraic turbulence model was used for the first set of runs. Additional runs were then made with the Chien $k-\varepsilon$ model, using the converged Baldwin-Lomax results as initial conditions. For the Baldwin-Lomax calculation, DTCAP was set equal to 5.0 for the first 800 time steps, 1.0 for the next 800 steps, and 0.5 for the rest of the calculation. For DTCAP $\geq 1.0$, the actual time step was computed by the code to limit the maximum change in density or pressure to $10 \%$, the value specified by PCQMAX. A total of 14,400 time steps were taken. The Chien $k-\varepsilon$ calculation also used a DTCAP value of 0.5 , for 7500 additional time steps.

Figure 11 shows computational "oil-flow" patterns on the flat plate. ${ }^{6}$ Starting at the wedge leading edge station, "particles" were released at every grid point along the outer $z$ boundary, selected grid points along a line at $x=0$, and at every grid point along a line near the inner $z$ boundary.

6. Computationally, these are actually in the plane of the first grid point above the surface, since all the velocities on the surface are zero. 


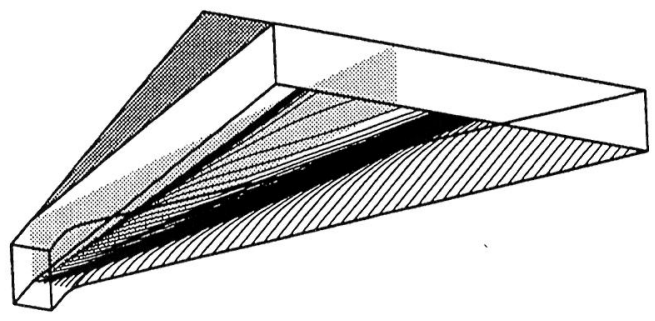

Figure 11. Computational "oil-flow" patterns, glancing shock/turbulent boundary layer interaction.

The computed values of the skin friction coefficient, the surface streamline angle, and the static pressure are compared with experimental data reported by Settles and Dodson (1991) in Figures 12(a) through 12(c). Computed results are shown for both the BaldwinLomax and Chien $k-\varepsilon$ calculations. The skin friction data were obtained using a laser interferometer skin friction meter, which measures the thickness of an oil film on the surface (Kim and Settles, 1990). The error bars in the skin friction plot are those reported by Settles and Dodson (1991), and reflect the repeatability of the measurement. The experimental surface streamline angles were measured from kerosene-lampblack flow visualization patterns, with an accuracy of $\pm 5 \%$. The static pressure data were measured using taps on the surface, and are believed accurate to within $\pm 3 \%$. Data points without error bars (and this includes all the surface streamline angle and pressure data) have errors comparable to, or less than, the size of the symbols in the plot.

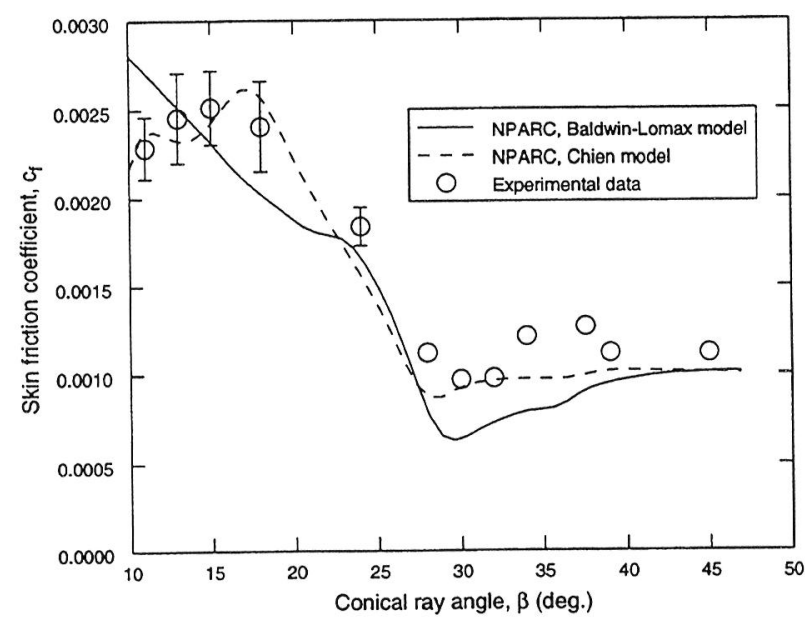

(a) Skin friction coefficient

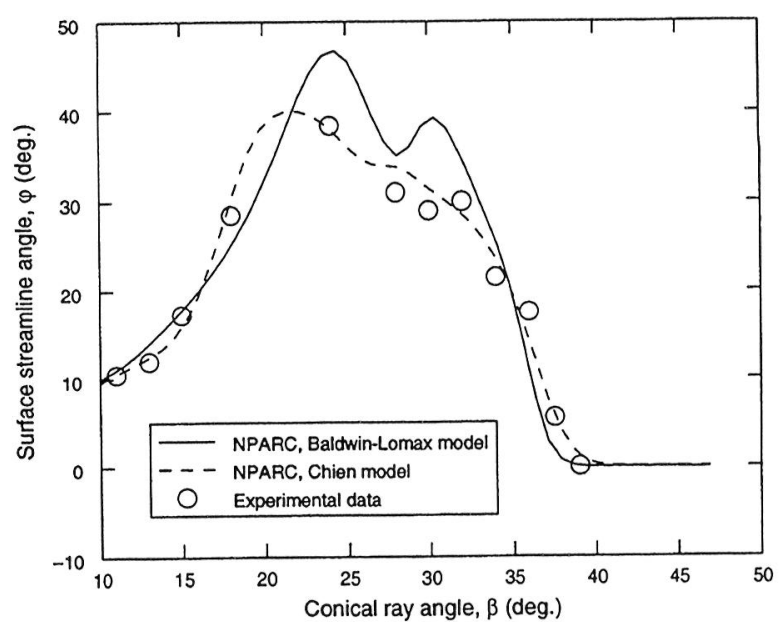

(b) Surface streamline angle

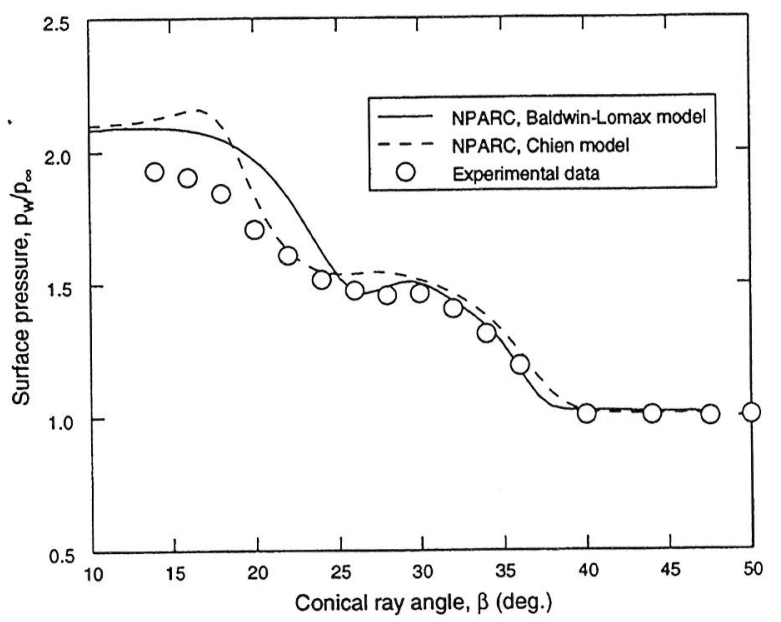

(c) Static pressure

Figure 12. Computed results compared with experimental data, glancing shock/turbulent boundary layer interaction.

\section{Additional Validation Cases}

Work is currently planned or underway on a variety of additional model and example validation cases. Model validation studies currently scheduled to be run over the next two years include:

- Turbulent flow past a flat plate with and without heat transfer

- Subsonic diffuser flow (Dudek, Georgiadis, and Yoder, 1996)

- Subsonic flow past an airfoil

- Glancing shock wave/turbulent boundary layer interaction

- Rearward facing step

- Subsonic flow through an S-duct

- Supersonic free jet flow 
Selected cases from some of the above studies will also be documented as example validation cases. Additional example cases currently scheduled include:

- External flow past an aft boat-tail configuration

- Unsteady normal shock, in conjunction with a NASA LeRC experiment

- Transition duct

- Counter-flowing jet

- Crossed glancing shocks with and without bleed, in conjunction with a NASA LeRC experiment

\section{Data Archive}

For validation cases to be of maximum benefit to the user community, the results must be made readily available. The information should be also be detailed enough to permit the calculations to be repeated with relative ease by independent code users. A formal electronic archive system has been established to meet these criteria.

This archive is the central repository for the model validation cases, example validation cases, and check cases computed by and for the NPARC Alliance. It is intended for use by NPARC users and developers, and allows easy access to the results of latest validation studies. The archive includes all the input files used to run the cases, the output files, the experimental data used for comparison with computed results, and written documentation providing an overview of each case and a discussion of the results. The archive is accessible over the Internet via anonymous $\mathrm{ftp}$ at info.arnold.af.milpub/nparc, and from the existing NPARC WWW home page located at http://info.arnold.af.mil/nparc/index.html.

\section{References}

Baldwin, B. S., and Lomax, H. (1978) "Thin Layer Approximation and Algebraic Model for Separated Turbulent Flows," AIAA Paper 78-257.

Chien, K.-Y. (1982) "Predictions of Channel and Boundary-Layer Flows with a Low-Reynolds-Number Turbulence Model," AIAA Journal, Vol. 20, No. 1, pp. 33-38.

Coles, D. E., and Hirst, E. A. eds. (1968) Computation of Turbulent Boundary Layers - 1968, Proceedings of the AFOSR-IFP-Stanford Conference, Vol. II, Stanford University.

Daily, J. W., and Harleman, D. R. F. (1966) Fluid Dynamics, Addison-Wesley, Reading, Massachusetts.

Dudek, J. C., Georgiadis, N. J., and Yoder, D. A. (1996)

"Calculation of Turbulent Subsonic Diffuser Flows
Using the NPARC Navier-Stokes Code," AIAA Paper 96-0497.

Eggers, J. M. (1966) "Velocity Profiles and Eddy Viscosity Distributions Downstream of a Mach 2.22 Nozzle Exhausting to Quiescent Air," NASA TN D-3601.

Hodge, B. K., and Adams, J. C., Jr. (1978) "The Calculation of Compressible Transitional, Turbulent, and Relaminarization Boundary Layers over Smooth and Rough Surfaces using an Extended Mixing Length Hypothesis," AEDC-TR-77-96.

Kim, K.-S., and Settles, G. S. (1990) "Skin Friction Measurements by Laser Interferometry in Swept Shock/Boundary-Layer Interactions," AIAA Journal, Vol. 28, No. 1, pp. 133-139.

Kim, K.-S., Lee, Y., Alvi, F. S., Settles, G. S., and Horstman, C. C. (1991) "Skin Friction Measurements and Computational Comparison of Swept Shock/Boundary-Layer Interactions," AIAA Joumal, Vol. 29, No. 10, pp. 1643-1650.

Mehta, U. B. (1990) "Computational Requirements for Hypersonic Flight Performance Estimates," Journal of Spacecraft and Rockets, Vol. 27, No. 2, pp. 103-112.

NPARC Alliance (1994) “A User's Guide to NPARC Version 2.0."

Roberts, G. O. (1971) "Computational Meshes for Boundary Layer Problems," Proceedings of the Second International Conference on Numerical Methods in Fluid Dynamics, Lecture Notes in Physics, Vol. 8, Springer-Verlag, New York, pp. 171-177.

Settles, G. S., and Dodson, L. J. (1991) "Hypersonic Shock/Boundary-Layer Interaction Database," NASA CR 177577.

Steinbrenner, J. P., Chawner, J. R., and Fouts, C. R. (1990) "The GRIDGEN 3D Multiple Block Grid Generation System," WRDC-TR-90-3022.

Thomas, P. D. (1979) "Numerical Method for Predicting Flow Characteristics and Performance of Nonaxisymmetric Nozzles - Theory," NASA CR 3147.

White, F. M. (1974) Viscous Fluid Flow, McGraw-Hill Book Company, New York. 
Public reporting burden for this coltection of information is estimated to average 1 hour per response, including the time tor reviewing instructions, searching existing cata sources. gathering and maintaining the data neoded, and completing and reviowing the colbection of information. Send comments regarding this burden estimate of any other aspect of inis collection of intormation, including suggestions for

Davis Highway, Suite 1204, Arlington, VA 22202-4302, and to the Otfice of Management and Budget, Paperwork Reduction Project (0704-0188), Wash

\begin{tabular}{|l|c|c|c|}
\hline 1. AGENCY USE ONLY (Leave blank) & $\begin{array}{c}\text { 2. REPORT DATE } \\
\text { January } 1996\end{array}$ & $\begin{array}{r}\text { 3. REPORT TYPE AND DATES COVERED } \\
\text { Technical Memorandum }\end{array}$ \\
\hline 4. TMTLE AND SUBTTLE & $\begin{array}{r}\text { 5. FUNDING NUMBERS } \\
\hline\end{array}$
\end{tabular}

Results and Current Status of the NPARC Alliance Validation Effort

5. FUNDING NUMBERS

4. TITLE AND SUBTITLE

Results and Current Status of the NPARC Alliance Validation Effort

6. AUTHOR(S)

WU-505-62-52

Charles E. Towne and Ralph R. Jones

7. PERFORMING ORGANIZATION NAME(S) AND ADDRESS(ES)

8. PERFORMING ORGANIZATION REPORT NUMBER

National Aeronautics and Space Administration

Lewis Research Center

E-10064

Cleveland, Ohio 44135-3191

9. SPONSORINGMONITORING AGENCY NAME(S) AND ADDRESS(ES)

National Aeronautics and Space Administration

Washington, D.C. 20546-0001

10. SPONSORINGMONITORING AGENCY REPORT NUMBER

NASA TM-107134

AIAA-96-0387

11. SUPPLEMENTARY NOTES

Prepared for the 34th Aerospace Sciences Meeting and Exhibit sponsored by the American Institute of Aeronautics and Astronautics, Reno, Nevada, January 15-18, 1996. Charles E. Towne, NASA Lewis Research Center and Ralph R. Jones, Sverdrup Technology, Inc./ AEDC Group, Arnold Engineering Development Center, Arnold Air Force Base, Tennessee. Responsible person, Charles E. Towne, organization code 2660 , (216) 433-5851.

12a. DISTRIBUTIONAVAILABILTTY STATEMENT 12b. DISTRIBUTION CODE

Unclassified -Unlimited

Subject Category 34

This publication is available from the NASA Center for Aerospace Information, (301) 621-0390.

13. ABSTRACT (Maximum 200 words)

The NPARC Alliance is a partnership between the NASA Lewis Research Center (LeRC) and the USAF Amold Engineering Development Center (AEDC) dedicated to the establishment of a national CFD capability, centered on the NPARC Navier-Stokes computer program. The three main tasks of the Alliance are user support, code development, and validation. The present paper is a status report on the validation effort. It describes the validation approach being taken by the Alliance. Representative results are presented for laminar and turbulent flat plate boundary layers, a supersonic axisymmetric jet, and a glancing shock/turbulent boundary layer interaction. Cases scheduled to be run in the future are also listed. The archive of validation cases is described, including information on how to access it via the Intemet.

\begin{tabular}{|c|c|c|}
\hline 14. SUBJECT TERMS \\
Navier-Stokes; CFD; Validation \\
\hline $\begin{array}{c}\text { 17. SECURTY CLASSIFICATION } \\
\text { OF REPORT } \\
\text { Unclassified }\end{array}$ & $\begin{array}{c}\text { 18. SECURTY CLASSIFICATION } \\
\text { OF THIS PAGE } \\
\text { Unclassified }\end{array}$ & $\begin{array}{c}\text { 19. SECURITY CLASSIFICATION } \\
\text { OF ABSTRACT } \\
\text { Unclassified }\end{array}$ \\
\hline
\end{tabular}


National Aeronautics and

Space Administration

Lewis Research Center

21000 Brookpark Rd.

Cleveland, $\mathrm{OH}$ 44135-3191

Officlal Business

Penalty for Privale Use $\$ 300$

POSTMASTER: If Undeliverable - Do Not Return 